Rodrigo Pereira Martins

Renderização em tempo real
utilizando mapas de
iluminação em alta precisão

DISSERTAÇÃO DE MESTRADO

DEPARTAMENTO DE INFORMÁTICA

Programa de Pós-graduação em

Informática 

Pontifícia $U_{\text {Niversidade }}$ Católica $_{\text {Do Rio de Janeiro }}$

Rodrigo Pereira Martins

Renderização em tempo real utilizando mapas de iluminação em alta precisão

Dissertação apresentada como requisito parcial para obtenção do grau de Mestre pelo Programa de Pósgraduação em Informática do Departamento de Informática da PUC-Rio.

Orientador: Prof. Bruno Feijó 
Rodrigo Pereira Martins

\section{Renderização em tempo real utilizando mapas de iluminação em alta precisão}

Dissertação apresentada como requisito parcial para obtenção do grau de Mestre pelo Programa de Pósgraduação em Informática do Departamento de Informática do Centro Técnico Científico da PUC-Rio. Aprovada pela Comissão Examinadora abaixo assinada.

Prof. Bruno Feijó

Orientador

Departamento de Informática — PUC-Rio

Prof. Luiz Velho

Visgraf — Instituto de Matemática Pura e Aplicada

Prof. Waldemar Celes Departamento de Informática - PUC-Rio

Prof. José Eugenio Leal Coordenador Setorial do Centro Técnico Científico PUC-Rio 
Todos os direitos reservados. É proibida a reprodução total ou parcial do trabalho sem autorização da universidade, do autor e do orientador.

\section{Rodrigo Pereira Martins}

Graduou-se em Ciência da Computação pela UERJ (Universidade do Estado do Rio de Janeiro). Atualmente é pesquisador associado ao laboratório VisionLab ICAD IGAMES. Sua linha de pesquisa se concentra em computação gráfica em tempo real, algortimos de iluminação global e processamento de imagens. Trabalha no desenvolvimento de engines para jogos e jogos em diferentes plataformas

Ficha Catalográfica

Martins, Rodrigo P.

Renderização em tempo real utilizando mapas de iluminação em alta precisão/ Rodrigo Pereira Martins; orientador: Bruno Feijó - Rio de Janeiro : PUC-Rio, Departamento de Informática, 2006.

99 f. : il. ; $30 \mathrm{~cm}$

1. Dissertação (mestrado) - Pontifícia Universidade Católica do Rio de Janeiro, Departamento de Informática.

Inclui referências bibliográficas.

1. Informática - Teses. 2. Computação Gráfica. 3. Imagens High Dynamic Range. 4. Image Based Lighting. I. Feijó, Bruno. II. Pontifícia Universidade Católica do Rio de Janeiro. Departamento de Informática. III. Título. 


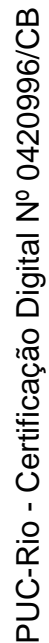

À Deus, minha mãe, meu pai, minha irmã, e todos aqueles de alguma forma me ajudam a percorrer esse caminho. 


\section{Agradecimentos}

Agradeço à minha família pelo suporte e carinho durante todos esses anos. Ao professor Bruno Feijó pela confiança depositada em mim e ao professor Luiz Velho pelos grandes ensinamentos durante a dissertação. Agradeço também aos meus amigos do Icad, especialmente: Lucas, Börje e Cris pelos grandes momentos de diversão no laboratório. Gostaria de agradecer a Bungie Studios por Halo, Halo 2, Halo 3 ... E finalmente à você que está lendo essa dissertação. Agradecimentos são devidos à CAPES pelo apoio financeiro, à FINEP pela infraestrutura e ao VisionLab por apoiar o desenvolvimento desta pesquisa. 


\title{
Resumo
}

\author{
Martins, Rodrigo P.; Feijó, Bruno. Renderização em tempo real \\ utilizando mapas de iluminação em alta precisão. Rio de \\ Janeiro, 2006. 99p. Dissertação de Mestrado — Departamento de \\ Informática, Pontifícia Universidade Católica do Rio de Janeiro.
}

Em 1997, o trabalho seminal de Paul Debevec e Jitendra Malik sobre a geração de imagens HDR (High Dynamic Range), a partir de câmeras comuns LDR (Low Dynamic Range), facilitou enormemente a geração de sondas de luz (light probes). Consequentemente, isto provocou uma explosão de trabalhos sobre renderização de objetos com imagens de luz do mundo real, que é conhecida como Iluminação Baseada em Imagem (ImageBased Lighting). A presente dissertação objetiva estudar esta nova área, procurando se situar na questão da composição (compositing) em tempo real de objetos sintéticos em imagens reais. Esta dissertação propõe um pipeline de renderização em tempo real para jogos 3D, no caso simples de cenas estáticas, adaptando a técnica não em tempo real apresentada por Paul Debevec em 1998. Não há trabalhos escritos na literatura sobre esta adaptação, embora haja alguma referência a desenvolvimentos realizados por fabricantes de placas gráficas nesta direção. Esta dissertação também apresenta um experimento com objetos difusos. Além do mais, o autor dá idéias na direção da solução de problemas de sombra para objetos difusos.

\section{Palavras-chave}

Imagens HDR, Processamento de imagens, Renderização em tempo real, Iluminação global. 


\section{Abstract}

Martins, Rodrigo P.; Feijó, Bruno. Real time rendering using high dynamic range illumination maps. Rio de Janeiro, 2006. 99p. MSc. Dissertation - Departamento de Informática, Pontifícia Universidade Católica do Rio de Janeiro.

In 1997, the seminal work by Paul Debevec and Jitendra Malik on the generation of HDR (High Dynamic Range) images, from ordinary LDR (Low Dynamic Range) cameras, facilitated the generation of light probes enormously. In consequence, this caused a boom of works on the rendering of objects with images of light from the real world, which is known as ImageBased Lighting. The present dissertation aims to study this new area, trying to situate itself in the question of real-time compositing of synthetic objects in real images. This dissertation proposes a real-time rendering pipeline for 3D games, in the simple case of static scenes, adapting the non-real-time technique presented by Paul Debevec in 1998. There is no written work about this adaptation in the literature, although exists some reference to developments done by graphics card manufacturers in this direction. Also this dissertation presents an experiment with diffuse objects. Moreover, the author gives ideas towards the solution of shadow problems for diffuse objects.

\section{Keywords}

HDR Images,Image processing, real time rendering, global illumination. 


\section{Conteúdo}

1 Introdução 13

1.1 O Problema da composição em HDR . . . . . . . . . . . . . . . . 13

1.2 Iluminação Baseada em Imagem . . . . . . . . . . . . . . . . . . . . . . . . . . . . . . . . . .

1.3 Imagens High Dynamic Range . . . . . . . . . . . . . . . . . . . . . 23

1.4 Objetivos e Organização da Dissertação . . . . . . . . . . . . . . . 30

2 Radiometria e fotometria 32

2.1 Luz como energia . . . . . . . . . . . . . . . . . . . . . . . . 32

2.1 .1 Medidas radiométricas . . . . . . . . . . . . . . . . . . . . . . . . . . 32

2.2 Medidas fotométricas . . . . . . . . . . . . . . . 35

3 O Tratamento HDR 38

3.1 Aquisição de imagens HDR . . . . . . . . . . . . . . . . . . . 38

3.1.1 Recuperação de curvas de resposta . . . . . . . . . . . . . 39

3.1.2 Criação de mapas de radiância . . . . . . . . . . . . . . 43

3.2 Renderização de objetos sintéticos utilizando mapas de iluminação HDR . . . . . . . . . . . . . . . . . . 44

3.2 .1 Método geral . . . . . . . . . . . . . . . . 45

3.2.2 Captura de iluminação ambiente . . . . . . . . . . . . . 47

3.2.3 Mapeando mapas de iluminação à cena . . . . . . . . . . . . . . 47

3.2 .4 Renderização da cena . . . . . . . . . . . . . . . . . . . . . . 48

3.3 Codificação de imagens HDR . . . . . . . . . . . . . . . . . . . . . . . . . . . . . . . . . . . . . .

3.3.1 Características de codificação HDR . . . . . . . . . . . . . . 49

3.3 .2 Formatos . . . . . . . . . . . . . . . 50

3.3.2.1 IEEE 96-bit TIFF . . . . . . . . . . . . . . 50

3.3.2.2 Formato RGBE . . . . . . . . . . . . . . . . 50

3.3 .3 Formato OpenEXR . . . . . . . . . . . . . . . . . 52

3.3.4 Formato para aplicações tempo real sem suporte a texturas em ponto flutuante . . . . . . . . . . . . . . 53

4 Técnicas de Renderização HDR $\quad 54$

4.1 Superfícies HDR . . . . . . . . . . . . . . . . . . . . . 54

4.2 Pipeline HDR . . . . . . . . . . . . . . . . . . . 55

4.2 .1 Renderização da cena . . . . . . . . . . . . . . . . . . . 57

4.2.2 Diminuição da superfície de renderização . . . . . . . . . . . . . . . 57

4.2 .3 Estágio de Bright Pass Filter . . . . . . . . . . . . . . . . . . . 59

4.2 .4 Glare . . . . . . . . . . . . . . . . . . . . . . 61

4.2 .5 Combinação . . . . . . . . . . . . . . . . . 66

4.2 .6 Tonemapping ....................... 66

5 Iluminação Baseada em Imagem $\quad 69$

5.1 Modelos de iluminação e sombreamento . . . . . . . . . . . . . 69

5.1 .1 lluminação . . . . . . . . . . . . . . . . . . . . . 69 
5.1.1.1 Componente difuso . . . . . . . . . . . . . . . . . . 70

5.1.1.2 Componente especular . . . . . . . . . . . . . . . 71

5.1.1.3 Componente ambiente . . . . . . . . . . . . . 72

5.1.1.4 Modelos de iluminação . . . . . . . . . . . . . . . . . . 73

5.1.2 Sombreamento . . . . . . . . . . . . . . . . . . 74

5.2 Mapas de ambiente como iluminação . . . . . . . . . . . . . . 75

5.2.1 Conceitos básicos de iluminação baseada em imagem . . . . . 77

5.2.1.1 Aquisição do mapa de iluminação . . . . . . . . . . . . . . . . 78

5.2.1.2 Modelagem da geometria da cena . . . . . . . . . . . 79

5.2.1.3 Mapeamento do mapa de iluminação na superfície do ambiente 79

5.2.1.4 Renderização da cena e pós processamento . . . . . . . . . 80

5.3 Formatos de mapas de iluminação . . . . . . . . . . . . . . . . . 81

5.3.1 Mapas esféricos . . . . . . . . . . . . . . . . . . . . . . 81

5.3.2 Mapas cúbicos . . . . . . . . . . . . . . . . . . . . . . . . 82

5.4 IBL em tempo real . . . . . . . . . . . . . . . . . . . . . 83

5.4.1 Enviroment mapping . . . . . . . . . . . . . . . . 83

5.4.2 Mapas de iluminação difusos . . . . . . . . . . . . . . 87

5.4.2.1 Oclusão de Ambiente . . . . . . . . . . . . . . . . . . . . 88

5.4.3 Mapas de ambiente dinâmicos . . . . . . . . . . . . 89

6 Conclusões e trabalhos futuros $\quad 92$

6.1 Resultados ........................... . . 92

6.2 Conclusôes . . . . . . . . . . . . . . . . . . . . 93

6.3 Trabalhos Futuros . . . . . . . . . . . . . . . . . . . 94

Referências Bibliográficas $\quad 95$ 


\section{Lista de Figuras}

1.1 Experiência pioneira de Gene Miller com mapa de reflexão fotográfico: (a) ambiente real; (b) bola reflexiva sendo fotografada; (c) cachorro renderizado com o mapa de reflexão. Extraído de G.Miller [1] . . . . . . . . . . . . . . . . . . . . .

1.2 Câmera HDR de escaneamento panorâmico SpheroCamHDR (26 F-stops, resolução 10.600 pixels horizontal por 5.300 pixels vertical). Imagem e informações retiradas de www.spheron.com [2] . . . . . . . . . . . . . . . . . .

1.3 Técnica de geração de sonda de luz de Debevec [53]. (a) câmera comum com esfera reflexiva (bolas industriais cromadas de 5 $\mathrm{cm}$ ou bolas ornamentais de jardim de $15-30 \mathrm{~cm}$, encontradas no varejo); (b) geração de mapa de radiância a partir de fotografias com aberturas diferentes da Grace Cathedral, São Francisco (c); (d) sonda de luz da Grace Cathedral, com variação dinâmica de 200000:1. Material extraído de Paul Debevec [3] . . . . . . . .

1.4 Composição de objetos sintéticos em fotografias usando sondas de luz HDR. (a) modelo geométrico do objeto sintético; (b) iluminação do objeto com fundo de floresta; (c) iluminação do objeto com fundo de museu. As sondas usadas estão indicadas no canto superior esquerdo. Material extraído de [3] . . . . . . . 18

1.5 Processo geral da técnica de lluminação Baseada em Imagem . 21

1.6 Geração da textura HDR da cena distante para ser usada na solução de iluminação global . . . . . . . . . . . . . . . . . 22

1.7 Imagem convencional. . . . . . . . . . . . . . . . . . 25

1.8 Imagem HDR. . . . . . . . . . . . . . . . . . . . . . . . . . . . 26

2.1 Irradiancia . . . . . . . . . . . . . . . . . 33

2.2 Saída radiante . . . . . . . . . . . . . . . . 34

2.3 Intensidade radiante . . . . . . . . . . . . . . . . . 34

2.4 Radiância . . . . . . . . . . . . . . . . . . . 35

2.5 Plotagem da curva $V(\lambda) \ldots \ldots \ldots$

3.1 Conjunto de imagens com exposição diferentes . . . . . . . . . 39

3.2 Método para adição de objetos sintéticos . . . . . . . . . . . 46

3.3 Formato RGBE . . . . . . . . . . . . . . . . . . . . . . 51

3.4 Codificação dos bits no formato OpenEXR. um bit para sinal, cinco para expoente e dez para mantissa . . . . . . . . . . 52

4.1 Pipeline HDR para renderização em tempo real . . . . . . . . . 56

4.2 Estágio de bright pass filter . . . . . . . . . . . . . . . . 60

4.3 Comparação de filtros gaussianos com valores de pixel não saturados . . . . . . . . . . . . . . . 62

4.4 Plotagem de curva gaussiana . . . . . . . . . . . . . . 62

4.5 Acesso as coordenadas de textura e seus respectivos pesos. . . 65 
4.6 Separabilidade de filtros gaussianos . . . . . . . . . . . 66

4.7 Combinação de gaussianas com diferentes raios aplicadas em uma mesma imagem. A imagem original é apresentada a esquerda, as imagens a direita são o resultado da aplicação do filtro gaussiano de raio unitário e da combinação de diferentes raios. . . . . . . . . . . . . . . 67

4.8 Imagens filtradas de diferentes tamanhos combinadas para gerar o resultado final . . . . . . . . . . . . . . . . 68

5.1 Interpretação geométrica do componente difuso . . . . . . . . . 71

5.2 Modelos de sombreamento. . . . . . . . . . . . . . . . 75

5.3 Cena iluminada por luzes sintéticas . . . . . . . . . . 76

5.4 Cena iluminada por mapa de iluminação . . . . . . . . . . . 77

5.5 light probes com diferentes exposições . . . . . . . . . . . . . 79

5.6 Mapa de iluminação cúbico . . . . . . . . . . . . . . . . . . 82

5.7 Acesso ao valor de radiância da cena distante (light probe) . . . 84

5.8 Cena renderizada pelo autor utilizando utilizando mapa de iluminação. . . . . . . . . . . . . . . . 86

5.9 Objeto renderizado pelo autor com mapa de iluminação difuso . 88 


\section{Lista de Tabelas}

1.1 Luminância para diferentes ambientes de iluminação. . . . . . . 23

2.1 Diferentes medidas de quantidades radiométricas . . . . . . . . 35

2.2 Diferentes medidas de quantidades fotométricas . . . . . . . 37

4.1 Desempenho baseado nas dimensões do render target . . . . . 59

6.1 Desempenho médio baseado no tamanho do render target . . . 93 